Journal of Algebraic Combinatorics 2 (1993), 111-121

(c) 1993 Kluwer Academic Publishers, Boston. Manufactured in The Netherlands.

\title{
Combinatorics of Maximal Minors
}

\author{
DAVID BERNSTEIN AND ANDREI ZELEVINSKY* \\ Department of Mathematics, Northeastern University, Boston, MA 02115
}

Received December 16, 1991; Revised November 10, 1992

\begin{abstract}
We continue the study of the Newton polytope $\prod_{m, n}$ of the product of all maximal minors of an $m \times n$-matrix of indeterminates. The vertices of $\prod_{m, n}$ are encoded by coherent matching fields $\Lambda=\left(\Lambda_{\sigma}\right)$, where $\sigma$ runs over all $m$-element subsets of columns, and each $\Lambda_{\sigma}$ is a bijection $\sigma \rightarrow[m]$. We show that coherent matching fields satisfy some axioms analogous to the basis exchange axiom in the matroid theory. Their analysis implies that maximal minors form a universal Gröbner basis for the ideal generated by them in the polynomial ring. We study also another way of encoding vertices of $\prod_{m, n}$ for $m \leq n$ by means of "generalized permutations", which are bijections between $(n-m+1)$-element subsets of columns and $(n-m+1)$-element submultisets of rows.
\end{abstract}

Keywords: matching field, Newton polytope, maximal minor

\section{Main results}

In this paper we continue the study of the Newton polytope $\prod_{m, n}$ of the product of all maximal minors of an $m \times n$ matrix of indeterminates, which had begun in [1]. This study has several algebraic-geometric and analytic motivations and applications, which were discussed in [1]. But the results and methods in this paper are mostly combinatorial, and the proofs use only a bit of convex geometry. Here we prove some of the conjectures made in [1] including Conjecture 5.7 (Theorem 1 below). As shown in [1, §7], this implies the following important property of maximal minors.

THEOREM 0. [1, Conjecture 7.1]. The set of all maximal minors of a generic $m \times n$ matrix $X=\left(x_{i j}\right)$ is a universal Gröbner basis for the ideal generated by them in the polynomial ring $\mathrm{C}\left[x_{i j}\right]$.

This paper is essentially self-contained and can be read independently of [1]. To state our main results we reiterate some terminology and notation from [1]. We fix two integers $m$ and $n$ with $2 \leq m \leq n$. Let $\mathbf{R}^{m \times n}$ be the space of real $m \times n$ matrices. We abbreviate $[n]:=\{1,2, \ldots, n\}$. Throughout the whole paper 
we identify and denote by the same symbol a subset $\Omega \subset[m] \times[n]$ of matrix indices and the corresponding indicator matrix $\sum_{(i, j) \in \Omega} E_{i j}$, where the $E_{i j}$ are matrix units.

For every m-element subset $\sigma \in[n]$ we call a matching with support $\sigma$ any bijection $\Lambda_{\sigma}: \sigma \rightarrow[m]$. By slight abuse of notation, we use the same symbol $\Lambda_{\sigma}$ for the graph of a matching $\left\{(i, j) \in[m] \times[n]: j \in \sigma, i=\Lambda_{\sigma}(j)\right\}$ (and also for its indicator matrix). A matching field of format $m \times n$ is a choice of a matching $\Lambda_{\sigma}$ for each $m$-element subset $\sigma \subset[n]$. Given any matching field $\Lambda=\left(\Lambda_{\sigma}\right)$, we let $v(\Lambda)=\sum_{\sigma} \Lambda_{\sigma}$ denote the sum of its indicator matrices. The polytope $\prod_{m, n}$ can be combinatorially defined as the convex hull in $\mathbf{R}^{m \times n}$ of the matrices $v(\Lambda)$ for all matching fields $\Lambda$ (see $[1, \S 1]$ ).

A matching field $\Lambda$ is called coherent if $v(\Lambda)$ is a vertex of the polytope $\prod_{m, n}$. By $[1, \S 1]$, coherent matching fields can be described as follows. Define the scalar product on $\mathbf{R}^{m \times n}$ by $\langle U, V\rangle=\sum_{i, j} u_{i j} v_{i j}$. Then a matching field $\Lambda=\left(\Lambda_{\sigma}\right)$ is coherent if and only if there exists a real matrix $\psi=\left(\psi_{i j}\right)$ such that $\left.\left\langle\psi, \Lambda_{\sigma}\right\rangle\right\rangle$ $\left\langle\psi, \Lambda_{\sigma}^{\prime}\right\rangle$ for every $\sigma$ and every matching $\Lambda_{\sigma}^{\prime}: \sigma \rightarrow[m]$ different from $\Lambda_{\sigma}$. In this case we say that $\psi$ supports $\Lambda$. The set of matrices $\psi$ which support $\Lambda$ is the normal cone of $\prod_{m, n}$ at the vertex $v(\Lambda)$.

It was shown in [1, Proposition 2.2] that every coherent matching field satisfies the following linkage axiom:

For every $i \in[m]$ and every $(m+1)$-element subset $\tau \subset[n]$ there exist two distinct $j, j^{\prime} \in \tau$ such that the matchings $\Lambda_{\tau \backslash j}$ and $\Lambda_{\tau \backslash j^{\prime}}$ agree outside the $i$ th row, i.e.,

$$
\Lambda_{\tau \backslash j}-E_{i j^{\prime}}=\Lambda_{\tau \backslash j^{\prime}}-E_{i j}
$$

Following $[1, \S 5]$ we associate to every matching field $\Lambda=\left(\Lambda_{\sigma}\right)$ and every subset $\rho \subset[n]$ of cardinality $n-m+1$ a mapping $\Omega_{\rho}: \rho \rightarrow[m]$ by the following rule:

$$
\Omega_{\rho}(j):=\Lambda_{\bar{\rho} \cup\{j\}}(j),
$$

where $\bar{\rho}:=[n] \backslash \rho$. As in the case of matchings, we use the same symbol $\Omega_{\rho}$ for its graph $\left\{(i, j) \in[m] \times[n]: j \in \rho, i=\Omega_{p}(j)\right\}$ (and also for its indicator matrix).

We are now in a position to state our first main result. We say that a subset $\Sigma \subset[m] \times[n]$ is transversal to a matching field $\Lambda=\left(\Lambda_{\sigma}\right)$ if $\Sigma \cap \Lambda_{\sigma} \neq \emptyset$ for all $\sigma$.

THEOREM 1. [1, Conjecture 5.7]. Let $\Lambda=\left(\Lambda_{\sigma}\right)$ be a matching field of format $m \times n$ satisfying the linkage axiom. Then a subset $\Sigma \subset[m] \times[n]$ is transversal to $\Lambda$ if and only if $\Sigma \supset \Omega_{\rho}$ for some $(n-m+1)$-element subset $\rho \subset[n]$.

As indicated in the introduction to [1], the linkage axiom is an analog to the basis exchange axiom in the theory of matroids. In the course of the proof of Theorem 1 we sharpen this analogy by presenting two other "matroid-like" characterizations of matching fields which satisfy the linkage axiom.

THEOREM 2. The following conditions on a matching field $\Lambda=\left(\Lambda_{\sigma}\right)$ are equivalent: 
(a) $\Lambda$ satisfies the linkage axiom.

(b) For every two distinct m-element subsets $\sigma, \sigma^{\prime} \subset[n]$ there exists $j^{\prime} \in \sigma^{\prime} \backslash \sigma$ with the following property: if $\Lambda_{\sigma^{\prime}}\left(j^{\prime}\right)=i$ and $j=\Lambda_{\sigma}^{-1}(i)$ then the matchings $\Lambda_{\sigma \backslash j \cup\left\{j^{\prime}\right\}}$ and $\Lambda_{\sigma}$ agree outside the ith row, i.e.,

$$
\Lambda_{\sigma \backslash j \cup\left\{j^{\prime}\right\}}-E_{i j^{\prime}}=\Lambda_{\sigma}-E_{i j} .
$$

(c) For every two distinct m-element subsets $\sigma, \sigma^{\prime}$ of [n] having a common column $j_{0}$ such that $\Lambda_{\sigma}\left(j_{0}\right) \neq \Lambda_{\sigma^{\prime}}\left(j_{0}\right)$ there exists an m-element subset $\sigma^{\prime \prime} \subset \sigma \cup \sigma^{\prime} \backslash j_{0}$ such that $\Lambda_{\sigma^{\prime \prime}} \subset \Lambda_{\sigma} \cup \Lambda_{\sigma^{\prime}}$.

Theorem 2 will allow us to prove the following converse of Theorem 1.

THEOREM 3. Let $\Lambda=\left(\Lambda_{\sigma}\right)$ be a matching field of format $m \times n$. Suppose that every minimal with respect to inclusion subset of $[m] \times[n]$ transversal to $\Lambda$ coincides with some $\Omega_{\rho}$. Then $\Lambda$ satisfies the linkage axiom.

Theorems $1-3$ will be proven in $\S 2$.

From now on we set $p:=n-m+1$. If $p=1$ i.e., $m=n$, then a matching field $\Lambda$ is simply a bijection between the set of columns and the set of rows of a square matrix. It was suggested in $[1, \S 6]$, that the vertices of $\prod_{m, n}$ for the general $m$ and $n$ can be encoded by some bijections between the sets of "generalized columns" and "generalized rows" of a rectangular matrix. To be more precise, let $C$ be the set of all $p$-element subsets of $[n]$, and $R$ the set of all nonnegative integer vectors $\alpha=\left(\alpha_{1}, \ldots, \alpha_{m}\right)$ with $\sum_{i} \alpha_{i}=p$. Clearly, $C$ and $R$ have the same cardinality $\left(\begin{array}{l}n \\ p\end{array}\right)=\left(\begin{array}{c}n \\ m-1\end{array}\right)$. Every matching field $\Lambda$ gives rise to a mapping $w=w_{A}: C \rightarrow R$ defined by

$$
w(\rho)_{i}:=\operatorname{card}\left(\Omega_{\rho}^{-1}(i)\right)
$$

THEOREM 4. If $\Lambda$ is a coherent matching field then $w_{\Lambda}: C \rightarrow R$ is a bijection.

This result establishes Conjecture 6.11 from [1] for coherent matching fields.

THEOREM 5. A coherent matching field $\Lambda$ is uniquely determined by the bijection $w_{\Lambda}: C \rightarrow R$.

We will give two different proofs of Theorem 5 . The first one shows that for every matching field $\Lambda$ (not necessarily coherent) the point $v(\Lambda):=\sum_{\sigma} \Lambda_{\sigma} \in \prod_{m, n}$ is recovered from the mapping $w_{A}: C \rightarrow R$ as follows:

We identify a subset $\rho \subset[n]$ with its indicator vector $\left(\rho_{1}, \ldots, \rho_{n}\right)$, where $\rho_{j}=1$ if $j \in \rho$, and $\rho_{j}=0$ if $j \notin \rho$. For every $\alpha \in R, \rho \in C$ we denote by $\alpha \cdot \rho$ an $m \times n$ matrix whose $(i, j)$-th entry is equal to $\alpha_{i} \rho_{j}$. We associate to a mapping $w: C \rightarrow R$ the matrix 


$$
M(w):=\sum_{\rho \in C} w(\rho) \cdot \rho .
$$

Let $\mathbf{1}_{m \times n}$ denote an $m \times n$ matrix with all entries equal to 1 .

THEOREM 6. Let $\Lambda$ be an arbitrary matching field (not necessarily coherent), and $w_{A}: C \rightarrow R$ be the corresponding mapping. Then

$$
v(\Lambda)=M\left(w_{\Lambda}\right)-\left(\begin{array}{c}
n-1 \\
m
\end{array}\right) \mathbf{1}_{m \times n}
$$

In the second proof of Theorem 5 we assume that $\Lambda$ is coherent and show that all the sets $\Omega_{\rho}$ can be recovered by the bijection $w_{A}: C \rightarrow R$. The set $R$ is a set of all integer points of a "thick" simplex with vertices $p e_{1}, p e_{2}, \ldots, p e_{m}$, where $e_{1}, \ldots, e_{m}$ are standard basis vectors in $\mathbf{R}^{m}$. We make $R$ a graph with $\alpha$ and $\alpha^{\prime}$ joined by an edge if and only if $\alpha-\alpha^{\prime}=e_{i}-e_{i^{\prime}}$ for some indices $i \neq i^{\prime}$. By a path from $\alpha$ to $\alpha^{\prime}$ we mean a chain $\left(\alpha(0)=\alpha, \alpha(1), \ldots, \alpha(d)=\alpha^{\prime}\right)$ of minimal possible length such that $\alpha(k-1)$ and $\alpha(k)$ are joined by an edge for $k=1, \ldots, d$; here $d$ is the distance between $\alpha$ and $\alpha^{\prime}$ in $R$.

THEOREM 7. Let $w=w_{\Lambda}: C \rightarrow R$ be a bijection corresponding to a coherent matching field $\Lambda$. Let $\rho \in C, i \in[m]$, and let $(\alpha(0), \alpha(1), \ldots, \alpha(d))$ be an arbitrary path from $w(\rho)$ to $p e_{i}$ in $R$. Then

$$
\Omega_{\rho}^{-1}(i)=\cap_{k=0}^{d} w^{-1}(\alpha(k)) .
$$

Theorem 7 provides some necessary conditions for a bijection $w: C \rightarrow R$ to be of the form $w_{\Lambda}$ for a coherent matching field $\Lambda$. Namely, the RHS of (7) must be independent of the choice of a path $(\alpha(0), \alpha(1), \ldots, \alpha(d))$, and the subsets $\Omega_{\rho}^{-1}(i)$ defined by means of (7) must satisfy

$$
\operatorname{card}\left(\Omega_{\rho}^{-1}(i)\right)=w(\rho)_{i}, \Omega_{\rho}^{-1}(i) \cap \Omega_{\rho}^{-1}\left(i^{\prime}\right)=\emptyset
$$

for all $\rho \in C$ and distinct $i, i^{\prime} \in[m]$. It turns out that these necessary conditions are sufficient in the case $n=m+1$. More precisely, we have the following theorem.

THEOREM 8. Suppose that $n=m+1$, i.e., $p=2$ Then the following conditions on a bijection $u: R \rightarrow C$ are equivalent :

(a) $A$ bijection $u$ has the form $w_{\Lambda}^{-1}$ for some coherent matching field $\Lambda$ of format $m \times(m+1)$

(b) For every two distinct $i, i^{\prime} \in[m]$

$$
\operatorname{card}\left(u\left(2 e_{i}\right) \cap u\left(e_{i}+e_{i^{\prime}}\right)\right)=1, u\left(2 e_{i}\right) \cap u\left(e_{i}+e_{i^{\prime}}\right) \cap u\left(2 e_{i^{\prime}}\right)=\emptyset .
$$


It would be interesting to investigate how far the conditions (8) are from being sufficient for general $m$ and $n$.

As a by-product of our proof of Theorem 4 we will derive the following alternative description of the polytope $\prod_{m, n^{n}}$. For each nonnegative integer vector $\beta=\left(\beta_{1}, \ldots, \beta_{m}\right)$ with sum $n$ let $\Xi_{\beta}$ denote the polytope of nonnegative $m \times n$ matrices having row sums $\beta_{1}, \ldots, \beta_{m}$ and all column sums equal to 1 .

THEOREM 9. The polytope $\prod_{m, n}$ coincides with the Minkowski sum $\sum_{\beta} \Xi_{\beta}$, the summation over all integer vectors $\beta=\left(\beta_{1}, \ldots, \beta_{m}\right)$ with $\sum_{i} \beta_{i}=n$ and all $\beta_{i} \geq 1$.

Theorems 4-9 will be proven in $\S 3$.

\section{Proof of Theorems 1-3}

Proof of Theorem 2. We will use two equivalent versions of the linkage axiom established in [1].

Lemma 10. [1, Theorem 2.4 and Proposition 2.13]. The following conditions on a matching field $\Lambda=\left(\Lambda_{\sigma}\right)$ are equivalent:

(a) 1 satisfies the linkage axiom.

$\left(a^{\prime}\right)$ For every $(m+1)$-element subset $\tau \subset[n]$ there exists a tree $T$ with the set of vertices $\tau$ and the set of edges labeled bijectively by $[\mathrm{m}]$, such that for every $j_{0} \in \tau$ the matching $\Lambda_{\tau \backslash j_{0}}$ sends each $j \in \tau \backslash j_{0}$ to an index $i$ such that the unique path from $j$ to $j_{0}$ in $T$ starts with the edge labeled by $i$.

$\left(a^{\prime \prime}\right)$ For every $(m+1)$-element subset $\tau \subset[n]$ and any three different elements $j_{1}$, $j_{2}, j_{3} \in \tau:$ if $\Lambda_{\tau \backslash j_{1}}\left(j_{2}\right) \neq \Lambda_{\tau \backslash j_{3}}\left(j_{2}\right)$ then $\Lambda_{\tau \backslash j_{1}}\left(j_{3}\right)=\Lambda_{\tau \backslash j_{2}}\left(j_{3}\right)$.

To prove Theorem 2 we will establish the implications $\left(a^{\prime}\right) \Rightarrow(b) \Rightarrow(c) \Rightarrow\left(a^{\prime \prime}\right)$.

Proof of $\left(a^{\prime}\right) \Rightarrow(b)$. Let $\Lambda=\left(\Lambda_{\sigma}\right)$ be a matching field satisfying $\left(a^{\prime}\right)$.

Lemma 11. [1, Proposition 5.6]. Each subset $\Omega_{\rho}$ in (2) is transversal to $\Lambda$

In order to prove that $\Lambda$ satisfies (b) we choose two distinct $m$-element subsets $\sigma, \sigma^{\prime} \subset[n]$. We have to show that there exists $j^{\prime} \in \sigma^{\prime} \backslash \sigma$ which satisfies (3). Choose arbitrary $j_{0} \in \sigma \backslash \sigma^{\prime}$ and consider the $(n-m+1)$-element set $\rho:=\bar{\sigma} \cup\left\{j_{0}\right\}$, where $\bar{\sigma}=[n] \backslash \sigma$. By Lemma $11, \Omega_{\rho} \cap \Lambda_{\sigma^{\prime}} \neq \emptyset$. Choose a point $\left(i, j^{\prime}\right) \in \Omega_{\rho} \cap \Lambda_{\sigma^{\prime}}$. Now take $\tau:=\sigma \cup\left\{j^{\prime}\right\}$ and consider the tree $T$ provided by $\left(a^{\prime}\right)$. By definition of $\Omega_{\rho}$, we have $A_{\tau \backslash j_{0}}\left(j^{\prime}\right)=i$; therefore, the edge $i$ in $T$ passes through the vertex $j^{\prime}$. Let $j$ be the second end of this edge. Again using $\left(a^{\prime}\right)$ we see that $\Lambda_{\tau \backslash j^{\prime}}(j)=i$, and that $\Lambda_{\tau \backslash j}$ and $\Lambda_{\tau \backslash j^{\prime}}$ agree with each other 
outside the $i$ th row. But this is exactly the property (3) because $\tau \backslash j^{\prime}=\sigma$ and $\tau \backslash j=\sigma \backslash j \cup\left\{j^{\prime}\right\}$.

Proof of $(b) \Rightarrow(c)$. We proceed by induction on $p\left(\sigma, \sigma^{\prime}\right):=\operatorname{card}\left(\Lambda_{\sigma^{\prime}} \backslash \Lambda_{\sigma}\right)$. Clearly, $p\left(\sigma, \sigma^{\prime}\right) \geq 2$, so we start with the case when $p\left(\sigma, \sigma^{\prime}\right)=2$. Then the set $\tau:=\sigma \cup \sigma^{\prime}$ consists of $m+1$ elements, and we have $\sigma=\tau \backslash j^{\prime}, \sigma^{\prime}=\tau \backslash j$ for some $j, j^{\prime} \in \tau$, and $\Lambda_{\sigma}(k)=\Lambda_{\sigma^{\prime}}(k)$ for $k \in \tau \backslash\left\{j_{0}, j, j^{\prime}\right\}$. Applying the property (b) to the subsets $\sigma$ and $\sigma^{\prime}$ and taking into account that in this case $\sigma^{\prime} \backslash \sigma$ consists of one element $j^{\prime}$, we obtain that $\Lambda_{\tau \backslash j_{0}}\left(j^{\prime}\right)=\Lambda_{\sigma^{\prime}}\left(j^{\prime}\right)$, and $\Lambda_{\tau \backslash j_{0}}(k)=\Lambda_{\sigma}(k)$ for $k \in \sigma \backslash j_{0}$. Therefore, the subset $\sigma^{\prime \prime}:=\tau \backslash j_{0}$ satisfies (c), as desired.

Now suppose that $p\left(\sigma, \sigma^{\prime}\right) \geq 3$, and assume that (c) holds for all pairs $\sigma_{0}, \sigma^{\prime}$ with $p\left(\sigma_{0}, \sigma^{\prime}\right)<p\left(\sigma, \sigma^{\prime}\right)$. Apply (b) to $\sigma$ and $\sigma^{\prime}$, and let $j^{\prime} \in \sigma^{\prime} \backslash \sigma, j \in \sigma, i \in[m]$ be the elements satisfying (3). Set $\sigma_{0}:=\sigma \backslash j \cup\left\{j^{\prime}\right\}$. By (3), $\Lambda_{\sigma_{0}}\left(j^{\prime}\right)=i=\Lambda_{\sigma^{\prime}}\left(j^{\prime}\right)$, and $\Lambda_{\sigma_{0}}(k)=\Lambda_{\sigma}(k)$ for $k \in \sigma \backslash j$. This implies, in particular, that $\Lambda_{\sigma_{0}} \subset \Lambda_{\sigma} \cup \Lambda_{\sigma^{\prime}}$. If $j=j_{0}$ then the subset $\sigma^{\prime \prime}=\sigma_{0}$ satisfies (c), and we are done; so assume that $j \neq j_{0}$. By construction, $p\left(\sigma_{0}, \sigma^{\prime}\right)<p\left(\sigma, \sigma^{\prime}\right)$, so by inductive assumption we can find $\sigma^{\prime \prime} \subset \sigma_{0} \cup \sigma^{\prime} \backslash j_{0}$ such that $\Lambda_{\sigma^{\prime \prime}} \subset \Lambda_{\sigma_{0}} \cup \Lambda_{\sigma^{\prime}}$. But then $\Lambda_{\sigma^{\prime \prime}} \subset \Lambda_{\sigma} \cup \Lambda_{\sigma^{\prime}}$, and we are done.

To complete the proof of Theorem 2 it remains to observe that the property $\left(a^{\prime \prime}\right)$ is a special case of (c) for $\sigma=\tau \backslash j_{1}, \sigma^{\prime}=\tau \backslash j_{3}, j_{0}=j_{2}$.

Proof of Theorem 1. Let $\Lambda=\left(\Lambda_{\sigma}\right)$ be a matching field which satisfies the linkage axiom, and hence, by Theorem 2 , also the property (c). Taking into account Lemma 11 we have only to prove the following statement: If $\Sigma \subset[m] \times[n]$ is transversal to $\Lambda$ then $\Sigma \supset \Omega_{\rho}$ for some $(n-m+1)$-element subset $\rho \subset[n]$. Without loss of generality we can assume that $(m, n) \in \Sigma$ and $\Sigma$ is minimal transversal to $\Lambda$ with respect to inclusion. By minimality of $\Sigma$, there exists an $m$-element subset $\sigma \subset[n]$ such that

$$
\Sigma \cap \Lambda_{\sigma}=\{(m, n)\}
$$

Consider the restriction of $\Lambda$ to $[m] \times[n-1]$, i.e., the matching field $\Lambda^{\prime}$ of format $m \times(n-1)$ formed by all matchings $\Lambda_{\sigma^{\prime}}$ with $\sigma^{\prime} \subset[n-1]$. Let $\left(\Omega_{\alpha^{\prime}}\right)$ be the corresponding family of subsets of $[m] \times[n-1]$ constructed from $\Lambda^{\prime}$ by means of (2); here $\rho^{\prime}$ runs over $(n-m)$-element subsets of $[n-1]$. Let $\Sigma^{\prime}=\Sigma \cap([m] \times[n-1])$. Then $\Sigma^{\prime}$ is transversal to $\Lambda^{\prime}$. Using induction on $n$ we can assume that $\Sigma^{\prime} \supset \Omega_{\rho^{\prime}}$ for some $\rho^{\prime} \subset[n-1]$ (as a first inductive step we can take $n=m$, in which case our statement becomes tautological).

Set $\Omega:=\Omega_{p^{\prime}} \cup\{(m, n)\}$. We claim that $\Omega$ is transversal to $\Lambda$. By Lemma 11, $\Omega$ is transversal to $\Lambda_{\sigma^{\prime}}$ for all $\sigma^{\prime} \subset[n-1]$. It remains to prove that $\Omega \cap \Lambda_{\sigma^{\prime}} \neq \emptyset$ for each $\sigma^{\prime}$ containing $n$ and such that $\Lambda_{\sigma^{\prime}}(n) \neq m$. To show this we apply the property (c) from Theorem 2 to $\sigma, \sigma^{\prime}$ and $j_{0}=n$. We obtain that there exists $\sigma^{\prime \prime} \subset([n-1] \cap \sigma) \cup \sigma^{\prime}$ such that $\Lambda_{\sigma^{\prime \prime}} \subset \Lambda_{\sigma} \cup \Lambda_{\sigma^{\prime}}$. We have already seen that $\Omega \cap \Lambda_{\sigma^{\prime \prime}} \neq \emptyset$, therefore 


$$
\Omega \cap\left(\left(([m] \times[n-1]) \cap \Lambda_{\sigma}\right) \cup \Lambda_{\sigma^{\prime}}\right) \neq \emptyset .
$$

But $\Omega \cap\left(([m] \times[n-1]) \cap \Lambda_{\sigma}\right)=\emptyset$ by $(10)$, so $\Omega \cap \Lambda_{\sigma^{\prime}} \neq \emptyset$, as claimed.

It remains to show that $\Omega=\Omega_{\rho}$ for $\rho:=\rho^{\prime} \cup\{n\}$. But this is clear because for every $j \in \rho$ the matching $\Lambda_{\bar{p} \cup\{j\}}$ can intersect $\Omega$ only in the element $\Lambda_{\bar{p} \cup\{j\}}(j)$ (cf. [1, Lemma 5.4]). Theorem 1 is proven.

Proof of Theorem 3. Suppose that a matching field $\Lambda$ does not satisfy the linkage axiom. Then the property (c) of Theorem 2 also fails, i.e., there exist two $m$ element subsets $\sigma$ and $\sigma^{\prime}$ of $[n]$ and an index $j \in \sigma \cap \sigma^{\prime}$ such that $\Lambda_{\sigma}(j) \neq \Lambda_{\sigma^{\prime}}(j)$, and that there is no matching $\Lambda_{\sigma^{\prime \prime}}$ contained in $\Lambda_{\sigma} \cup \Lambda_{\sigma^{\prime}} \backslash([m] \times\{j\})$. Let $i=\Lambda_{\sigma}(j), i^{\prime}=\Lambda_{\sigma^{\prime}}(j)$, and

$$
\Sigma:=([m] \times[n]) \backslash\left(\Lambda_{\sigma} \cup \Lambda_{\sigma^{\prime}}\right) \cup([m] \times\{j\}) .
$$

Our choice of $\sigma$ and $\sigma^{\prime}$ implies that $\Sigma$ is transversal to $\Lambda$. Choose a subset $\Sigma_{0} \subset \Sigma$ which is minimal with respect to inclusion transversal to $\Lambda$. Since $\Sigma \cap \Lambda_{\sigma}=\{(i, j)\}, \Sigma \cap \Lambda_{\sigma^{\prime}}=\left\{\left(i^{\prime}, j\right)\right\}$, it follows that $\Sigma_{0}$ contains both $\{(i, j)\}$ and $\left\{\left(i^{\prime}, j\right)\right\}$. Therefore, $\Sigma_{0}$ is not one of the subsets $\Omega_{\rho}$, which proves Theorem 3.

\section{Proof of Theorems 4-9}

Proof of Theorem 4. We fix a coherent matching field $\Lambda=\left(\Lambda_{\sigma}\right)$; a matrix $\psi=\left(\psi_{i j}\right)$ supporting $\Lambda$; and a vector $\alpha=\left(\alpha_{1}, \ldots, \alpha_{m}\right) \in R$. Since $\operatorname{card}(R)=\operatorname{card}(C)$, to prove our theorem it is enough to construct $\rho \in C$ such that $w_{A}(\rho)=\alpha$.

For each nonnegative integer vector $\beta=\left(\beta_{1}, \ldots, \beta_{m}\right)$ with sum $n$, let $\Xi_{\beta}$ denote the polytope of nonnegative $m \times n$ matrices having row sums $\beta_{1}, \ldots, \beta_{m}$ and all column sums equal to 1 . Let $\Gamma_{\beta}$ be the vertex of $\Xi_{\beta}$ supported by $\psi$; we assume $\psi$ to be sufficiently generic so that $\Gamma_{\beta}$ is unique. Clearly, each $\Gamma_{\beta}$ is a $(0,1)$-matrix, and we identify it with its support which is a subset of $[m] \times[n]$, and also with the mapping $[n] \rightarrow[m]$ whose graph is this subset.

For each $i \in[m]$ let $\beta(i)$ denote the vector

$$
\beta(i)=\left(\alpha_{1}+1, \alpha_{2}+1, \ldots, \alpha_{i-1}+1, \alpha_{i}, \alpha_{i+1}+1, \ldots, \alpha_{m}+1\right) .
$$

LEMMA 12. For every two distinct indices $i, i^{\prime}=1, \ldots, m$ the ith row of $\Gamma_{\beta(i)}$ is contained in the ith row of $\Gamma_{\beta\left(i^{\prime}\right)}$.

Proof. We associate to $i$ and $i^{\prime}$ an edge-colored oriented graph $G_{i, i^{\prime}}$ with the vertex set $[m]$ and the color set $[n]$ as follows: There is an edge from $i_{1}$ to $i_{2}$ colored by $j$ whenever $i_{1} \neq i_{2},\left(i_{1}, j\right) \in \Gamma_{\beta(i)}$ and $\left(i_{2}, j\right) \in \Gamma_{\beta\left(i^{\prime}\right) \text {. Lemma }} 12$ is an immediate consequence of the following lemma. 
LEMMA 13. The graph $G_{i, i^{\prime}}$ is an oriented chain from $i^{\prime}$ to $i$.

Proof. First we show that $G_{i, i^{\prime}}$ has no oriented cycles. Suppose there is an oriented cycle

$$
i_{1} \stackrel{j_{1}}{\longrightarrow} i_{2} \stackrel{j_{2}}{\longrightarrow} \ldots \stackrel{j_{p-1}}{\longrightarrow} i_{p} \stackrel{j_{p}}{\longrightarrow} i_{1} .
$$

Consider two subsets $S=\left\{\left(i_{1}, j_{1}\right),\left(i_{2}, j_{2}\right), \ldots,\left(i_{p}, j_{p}\right)\right\}$ and $S^{\prime}=\left\{\left(i_{2}, j_{1}\right),\left(i_{3}, j_{2}\right)\right.$, $\left.\ldots,\left(i_{p}, j_{p-1}\right),\left(i_{1}, j_{p}\right)\right\}$ of $[m] \times[n]$. These two subsets occupy the same rows and columns, and we have $S \subset \Gamma_{\beta(i)}, S^{\prime} \subset \Gamma_{\beta\left(i^{\prime}\right)}$. But this contradicts the fact that both $\Gamma_{\beta(i)}$ and $\Gamma_{\beta\left(i^{i}\right)}$ are supported by the same linear functional $\psi$. Indeed, without loss of generality we can assume that $\langle\psi, S\rangle\left\langle\left\langle\psi, S^{\prime}\right\rangle\right.$. But then $\Gamma_{\beta(i)}+S^{\prime}-S$ is a point in the polytope $\Xi_{\beta(i)}$ with $\left.\left\langle\psi, \Gamma_{\beta(i)}+S^{\prime}-S\right\rangle\right\rangle\left\langle\psi, \Gamma_{\beta(i)}\right\rangle$, which contradicts our choice of $\Gamma_{\beta(i)}$.

We define the valency of a vertex $i_{0}$ in $G_{i, i^{\prime}}$ as the number of edges going out of $i_{0}$ minus the number of edges going into $i_{0}$. Since the graph describes passing from $\Gamma_{\beta(i)}$ to $\Gamma_{\beta\left(i^{\prime}\right)}$, all the vertices have valency 0 , except $i$ having valency -1 and $i^{\prime}$ having valency 1 . Since $G_{i, i^{\prime}}$ has no oriented cycles, it can be only a chain from $i^{\prime}$ to $i$. This completes the proof of Lemmas 12 and 13.

Now let $\rho(i):=\Gamma_{\beta(i)}^{-1}(i)$ be the set of columns occupied by the $i$ th row of $\Gamma_{\beta(i)}$, and let $\rho=\cup_{i=1}^{m} \rho(i)$. By Lemma 12, the subsets $\rho(i)$ are mutually disjoint. Since $\operatorname{card}(\rho(i))=\alpha_{i}$, it follows that $\operatorname{card}(\rho)=p$, i.e., $\rho \in C$.

Put $\Omega:=\cup_{i=1}^{m}(\{i\} \times \rho(i))$. To prove Theorem 4 it suffices to show that $\Omega=\Omega_{\rho}$, which implies that $w_{\Lambda}(\rho)=\alpha$.

LEMMA 14. There exists a tree $T$ with the vertex set $[m]$ whose edges are labeled bijectively by $\bar{\rho}:=[n] \backslash \rho$, such that:

(a) For every $i \in[m]$

$$
\Gamma_{\beta(i)}=\Omega \cup\left\{\left(i^{\prime}, j\right) \in[m] \times \bar{\rho}: i^{\prime} \stackrel{j}{\longrightarrow} i\right\} .
$$

(b) For every $j_{0} \in \rho_{i}$

$$
\Lambda_{\bar{p} \cup\left\{j_{0}\right\}}=\left\{\left(i, j_{0}\right)\right\} \cup\left\{\left(i^{\prime}, j\right) \in[m] \times \bar{\rho}: i^{\prime} \stackrel{j}{\longrightarrow} i\right\} ;
$$

here the notation " $i$ ' $\stackrel{j}{\longrightarrow} i$ " means that the unique path from $i^{\prime}$ to $i$ in $T$ starts with the edge labeled by $j$.

Proof.

(a) By Lemma 12, $\Omega \subset \Gamma_{\beta(i)}$ for each $i \in[m]$, and the difference $\Gamma_{\beta(i)} \backslash \Omega$ is the graph of a bijection $\Lambda_{[m] \backslash i}^{\prime}:[m] \backslash i \rightarrow \bar{\rho}$. Passing to transpose matrices, we 
represent the family of bijections $\left(\Lambda_{[m] \backslash i}^{\prime}\right), i=1, \ldots, m$ as a matching field of format $(m-1) \times m$ with the row set $\bar{\rho}$ and the column set $[m]$. This matching field is coherent because it is supported by the transpose of $\psi$. Therefore, it can be described as in Lemma $10\left(a^{\prime}\right)$, which is exactly our statement.

(b) Put $\sigma=\bar{\rho} \cup\left\{j_{0}\right\}$. Clearly, the RHS of (12) is (the graph of) a matching $\Lambda_{\sigma}^{\prime}: \sigma \rightarrow[m]$. By (a), $\Lambda_{\sigma}^{\prime} \subset \Gamma_{\beta(i)}$. It follows that $\Lambda_{\sigma}^{\prime}=\Lambda_{\sigma}$; otherwise, $\Gamma_{\beta(i)}-\Lambda_{\sigma}^{\prime}+\Lambda_{\sigma}$ would be a point of the polytope $\Xi_{\beta(i)}$ having a bigger value of the supporting form $\psi$ than $\Gamma_{\beta(i)}$.

Comparing (12) with (2), we see that if $j_{0} \in \rho(i)$ then $\Omega_{\rho}\left(j_{0}\right)=i$. Therefore, $\Omega=\Omega_{\rho}$, which completes the proof of Theorem 4 .

Proof of Theorem 9. Let $\psi$ be a generic linear form on the space of matrices. Let $\Lambda=\left(\Lambda_{\sigma}\right)$ be a coherent matching field supported by $\psi$, and for each nonnegative integer vector $\beta=\left(\beta_{1}, \ldots, \beta_{m}\right)$ with $\sum_{i} \beta_{i}=n$ and all $\beta_{i} \geq 1$, let $\Gamma_{\beta}$ be the vertex of $\Xi_{\beta}$ supported by $\psi$. It is enough to show that the vertex $v(\Lambda):=\sum_{\sigma} \Lambda_{\sigma}$ of $\prod_{m, n}$ coincides with the vertex $\sum_{\beta} \Gamma_{\beta}$ of $\sum_{\beta} \Xi_{\beta}$. This is a consequence of the following lemma.

LEMMA 15. For every $(i, j) \in[m] \times[n]$ there exists a bijection between the set of all m-element subsets $\sigma \subset[n]$ such that $j \in \sigma$ and $\Lambda_{\sigma}(j)=i$ and the set of all $\beta$ as above such that $(i, j) \in \Gamma_{\beta}$.

Proof. Choose $\sigma \ni j$ such that $\Lambda_{\sigma}(j)=i$. Take $\rho=\bar{\sigma} \cup\{j\} \in C$ and define $\alpha=w_{A}(\rho) \in R$. Let $\beta=\beta(i)$ be a vector associated to $\alpha$ by means of (11). Using the description of $\Gamma_{\beta}$ and $\Lambda_{\sigma}$ given by Lemma 14, we obtain the following relation:

$$
\Gamma_{\beta}=\Lambda_{\sigma}+\Omega_{\rho}-E_{i j}
$$

This implies that $(i, j) \in \Gamma_{\beta}$, and hence the mapping $\sigma \mapsto \beta$ is a desired bijection. Lemma 15 and Theorem 9 are proven.

Proof of Theorems 5 and 6. As usual, we will identify subsets of $[m] \times[n]$ with their indicator matrices. By definition,

$$
v(\Lambda)=\sum_{\sigma} \Lambda_{\sigma}=\sum_{\sigma} \sum_{j \in \sigma}\left\{\left(\Lambda_{\sigma}(j), j\right)\right\}
$$

Let us represent each one-element set $\left\{\left(\Lambda_{\sigma}(j), j\right)\right\}$ in the sum as a difference $\left\{\Lambda_{\sigma}(j)\right\} \times(\bar{\sigma} \cup\{j\})-\left\{\Lambda_{\sigma}(j)\right\} \times \bar{\sigma}$. We can write then $v(\Lambda)=M_{1}-M_{2}$, where

$$
M_{1}=\sum_{\sigma} \sum_{j \in \sigma}\left\{\Lambda_{\sigma}(j)\right\} \times(\bar{\sigma} \cup\{j\}), M_{2}=\sum_{\sigma} \sum_{j \in \sigma}\left\{\Lambda_{\sigma}(j)\right\} \times \bar{\sigma} .
$$


We will show that $M_{1}=M\left(w_{\Lambda}\right), M_{2}=\left(\begin{array}{c}n-1 \\ m\end{array}\right) \mathbf{1}_{m \times n}$.

If we put $\rho=\bar{\sigma} \cup\{j\}$, then by (2) we have $\Lambda_{\sigma}(j)=\Omega_{\rho}(j)$. Therefore, the summation for $M_{1}$ can be rewritten as

$$
M_{1}=\sum_{\rho \in C} \sum_{j \in \rho}\left\{\Omega_{\rho}(j)\right\} \times \rho .
$$

Remembering the definition of $w_{\Lambda}$ we see that the inner sum in (14) is $w_{\Lambda}(\rho) \cdot \rho$, so $M_{1}=M\left(w_{1}\right)$, as desired.

As for $M_{2}$, substituting $i=\Lambda_{\sigma}(j)$ we can rewrite the summation as

$$
M_{2}=\sum_{i, \sigma}\{i\} \times \bar{\sigma}
$$

Therefore, for every $i$ and $j$ the $(i, j)$-th entry of $M_{2}$ is equal to the number of $(n-m)$-element subsets $\bar{\sigma} \subset[n]$ which contain $j$, i.e., to $\left(\begin{array}{c}n-1 \\ n-m-1\end{array}\right)=\left(\begin{array}{c}n-1 \\ m\end{array}\right)$. This completes the proof of Theorem 6 . To prove Theorem 5 it remains to observe that a coherent matching field $\Lambda$ is uniquely determined by the vertex $v(\Lambda)$.

Proof of Theorem 7. Let $\alpha=\left(\alpha_{1}, \ldots, \alpha_{m}\right)=w_{A}(\rho)$. Then the distance $d$ from $\alpha$ to $p e_{i}$ is equal to $p-\alpha_{i}$, and the first vector in a path from $\alpha$ to $p e_{i}$ has the form $\alpha(1)=\alpha+e_{i}-e_{i^{\prime}}$ for some $i^{\prime} \neq i$. Let $\rho^{\prime}=w_{\Lambda}^{-1}(\alpha(1))$. By induction on $d$, to prove Theorem 7 it is enough to show that

$$
\Omega_{\rho}^{-1}(i)=\rho \cap \Omega_{\rho^{\prime}}^{-1}(i)
$$

Let $\beta(1), \ldots, \beta(m)$ be a family of vectors associated to $\alpha$ by means of (11). By (13), $\Omega_{\rho}^{-1}(i)$ is the set of columns occupied by the $i$ th row of $\Gamma_{\beta(i)}$. Now apply the same statement to a family $\beta^{\prime}(1), \ldots, \beta^{\prime}(m)$ associated by the same rule (11) to the vector $\alpha(1)$. By definition, $\beta^{\prime}(i)=\beta\left(i^{\prime}\right)$, so we obtain that $\Omega_{\beta^{\prime}}^{-1}(i)$ is the set of columns occupied by the $i$ th row of $\Gamma_{\beta\left(i^{\prime}\right)}$. Now (15) follows at once from the description of $\Gamma_{\beta(i)}$ and $\Gamma_{\beta\left(i^{\prime}\right)}$ given by Lemma 14 . Theorem 7 is proven.

Proof of Theorem 8. The implication $(a) \Rightarrow(b)$ is clear because for $n=m+1$, the condition (9) is simply another way of saying (8). So we suppose that a bijection $u: R \rightarrow C$ satisfies (9), and we wish to show that $u=w_{\Lambda}^{-1}$ for some coherent matching field $\Lambda$ of format $m \times(m+1)$.

Consider the edge-colored graph $T$ with the set of vertices $[m+1]$ and the set of colors $[m]$, where two vertices $j$ and $j^{\prime}$ are joined by an edge colored with $i$ whenever $u\left(2 e_{i}\right)=\left\{j, j^{\prime}\right\}$. Clearly, $T$ has $m$ edges, each color appearing exactly once.

LEMMA 16. Suppose that $s \geq 2$, and $j_{1}, j_{2}, \ldots, j_{s}$ are distinct elements of $[m+1]$ such that $j_{k}$ and $j_{k+1}$ are joined in $T$ by an edge colored with $i_{k}$ for $k=1, \ldots, s-1$. Then 


$$
u\left(e_{i_{k}}+e_{i_{k^{\prime}}}\right)=\left\{j_{k}, j_{k^{\prime}+1}\right\}
$$

for all $1 \leq k \leq k^{\prime} \leq s-1$.

Proof of Lemma 16. We proceed by induction on $s$. For $s=2$ the equality (16) is simply the definition of the graph $T$, and for $s=3$ it follows at once from (9). So we can assume that $s \geq 4$, and that (16) holds for all pairs $\left(k, k^{\prime}\right)$ except $(1, s-1)$. By (9), the two-element set $u\left(e_{i_{1}}+e_{i_{s-1}}\right)$ has nonempty intersection with each of the sets $u\left(2 e_{i_{1}}\right)=\left\{j_{1}, j_{2}\right\}$ and $u\left(2 e_{i_{R-1}}\right)=\left\{j_{s-1}, j_{s}\right\}$. Therefore, $u\left(e_{i_{1}}+e_{i_{s-1}}\right)$ has the form $\left\{j, j^{\prime}\right\}$, where $j \in\left\{j_{1}, j_{2}\right\}, j^{\prime} \in\left\{j_{s-1}, j_{s}\right\}$. But our inductive assumption implies that

$$
\left\{j_{1}, j_{s-1}\right\}=u\left(e_{i_{1}}+e_{i_{s-2}}\right),\left\{j_{2}, j_{s-1}\right\}=u\left(e_{i_{2}}+e_{i_{s-2}}\right),\left\{j_{2}, j_{s}\right\}=u\left(e_{i_{2}}+e_{i_{s-1}}\right) .
$$

Since $u$ is a bijection, the only opportunity left is $u\left(e_{i_{1}}+e_{i_{n-1}}\right)=\left\{j_{1}, j_{s}\right\}$, as desired.

By Lemma 16, every two vertices in $T$ are connected by at most one chain; hence, $T$ has no loops. Since $T$ has $m+1$ vertices and $m$ edges, we conclude that $T$ is a tree. Consider the matching field $\Lambda$ of format $m \times(m+1)$ associated to the tree $T$ as in Lemma $10\left(a^{\prime}\right)$. Then $\Lambda$ is coherent by [1, Theorem 2.4]. Comparing Lemma 16 with the formulas (2) and (4) above, we see that $u=w_{\Lambda}^{-1}$, which completes the proof of Theorem 8.

\section{Reference}

1. B. Sturmfels and A. Zelevinsky, "Maximal minors and their leading terms," Advances in Math, 98 (1993), 65-112. 